\title{
Design and characterization of a biodegradable double-layer scaffold aimed at periodontal tissue- engineering applications
}

\author{
João F. Requicha ${ }^{1,2,3}$, Carlos A. Viegas ${ }^{1,2,3}$, Shantesh Hede ${ }^{1,2}$, Isabel B. Leonor ${ }^{1,2}$, Rui L. Reis ${ }^{1,2}$ \\ and Manuela E. Gomes ${ }^{1,2 *}$ \\ ${ }^{1} 3 B$ 's Research Group, Headquarters of the European Institute of Excellence on Tissue Engineering and Regenerative Medicine, \\ University of Minho, Guimarães, Portugal \\ ${ }^{2}$ ICVS/3B's, PT Government Associated Laboratory, Braga/Guimarães, Portugal \\ ${ }^{3}$ Department of Veterinary Sciences, University of Trás-os-Montes e Alto Douro, Vila Real, Portugal
}

\begin{abstract}
The inefficacy of the currently used therapies in achieving the regeneration ad integrum of the periodontium stimulates the search for alternative approaches, such as tissue-engineering strategies. Therefore, the core objective of this study was to develop a biodegradable double-layer scaffold for periodontal tissue engineering. The design philosophy was based on a double-layered construct obtained from a blend of starch and poly- $\varepsilon$-caprolactone (30:70 wt\%; SPCL). A SPCL fibre mesh functionalized with silanol groups to promote osteogenesis was combined with a SPCL solvent casting membrane aiming at acting as a barrier against the migration of gingival epithelium into the periodontal defect. Each layer of the double-layer scaffolds was characterized in terms of morphology, surface chemical composition, degradation behaviour and mechanical properties. Moreover, the behaviour of seeded/cultured canine adipose-derived stem cells (cASCs) was assessed. In general, the developed double-layered scaffolds demonstrated adequate degradation and mechanical behaviour for the target application. Furthermore, the biological assays revealed that both layers of the scaffold allow adhesion and proliferation of the seeded undifferentiated cASCs, and the incorporation of silanol groups into the fibre-mesh layer enhance the expression of a typical osteogenic marker. This study allowed an innovative construct to be developed, combining a three-dimensional (3D) scaffold with osteoconductive properties and with potential to assist periodontal regeneration, carrying new possible solutions to current clinical needs. Copyright (C) 2013 John Wiley \& Sons, Ltd.
\end{abstract}

Received 24 June 2013; Revised 24 July 2013; Accepted 24 July 2013

Keywords scaffold; biodegradable; guided tissue regeneration; osteoconductive groups; periodontium; tissue engineering

\section{Introduction}

Periodontium, the organ that surrounds and sustains the tooth, is constituted by the alveolar bone, the cementum, the periodontal ligament (PDL) and the gingiva (Chen and Jin, 2010). The periodontium is often affected by periodontitis, an inflammatory disease highly prevalent in both humans and dogs, which can progresses with bone

*Correspondence to: M. E. Gomes, 3Bs Research Group, University of Minho, AvePark, S. Cláudio do Barco, 4860-909 Caldas das Taipas, Guimarães, Portugal. E-mail: megomes@dep.uminho.pt resorption, cementum necrosis and gingival recession or hyperplasia and ultimately, when untreated, leads to tooth exfoliation (Bosshardt and Sculean, 2009). Periodontitis has been identified as an important health problem, owing to its enormous prevalence and life-threatening implications on human and animal systemic health (Kuo et al., 2008).

Currently, there are several therapies used in clinical practice, namely the gingival flap techniques (Bosshardt and Sculean, 2009; Lin et al., 2008), scaling and root planning (Bosshardt and Sculean, 2009), root conditioning with demineralizing agents (Bosshardt and Sculean, 2009; Lin et al., 2008; Wikesjo and Nilveus, 1991), direct 
injection of growth or differentiation factors into the root surface [e.g. platelet-derived growth factor (PDGF), insulin growth factor-1 (IGF-1), bone morphogenetic proteins-2 and -7 (BMPs)] (Bosshardt and Sculean, 2009) or concentration of factors [platelet-rich plasma (PRP), enamel matrix derivatives (EMD)] (Chen and Jin, 2010; Tobita et al., 2008), in situ application of filler materials, such as autografts, allografts and alloplastic materials, viz. hydroxyapatite (HA) and tricalcium phosphate (TCP) (Wikesjo and Nilveus, 1991), and application of guided tissue regeneration (GTR) membranes.

GTR was proposed (Karring et al., 1993; Wikesjo and Nilveus, 1991) to selectively guide cells to proliferate in different compartments of the alveolar bone and PDL. This technique makes it possible to prevent gingival epithelium and connective tissue expansion, ankylosis and the radicular resorption phenomenon and, at the same time, to avoid collapse of the periodontal defect (Bosshardt and Sculean, 2009; Wikesjo et al., 2003).

The materials that have been used to produce GTR membranes are several: non-absorbable polymers, e.g. ePTFE (Wikesjo et al., 2003); absorbable polymers of synthetic origin, such as polylactic acid (PLA) (Park et al., 2000), poly (lactic-co-glycolic acid (PGLA) (Kurtis et al., 2002), polyglactin (Quinones et al., 1991) and biosynthetic cellulose (Galgut, 1990); or absorbable polymers of natural origin, such as collagen (Liao et al., 2005; Pitaru et al., 1989), cargile (Card et al., 1989) and chitosan (Ho et al., 2010; Mota et al., 2012). Besides those materials, composite membranes composed of PGLA with HA (Liao et al., 2005) or nano-apatite with poly( $\varepsilon$-caprolactone) (PCL) (Yang et al., 2009) have also been tested for this application. Other membranes have also been developed to work as vehicles for delivering biological molecules, such as growth factors (PDGF, BMPs, PRP) or antibiotics such as metronidazole (Kurtis et al., 2002), doxycycline (Chang and Yamada, 2000) or tetracycline (Park et al., 2000), in order to promote effective and aseptic cellular proliferation and differentiation in the desire tissue. In spite of all the therapeutic strategies developed to date for periodontal regeneration, there is still no treatment that can restore the functionality of all the damaged periodontal tissues. For this reason, clinicians tend to combine several of techniques mentioned above in attempts to reach the desired effect, resulting in complex treatments with unpredictable outcomes (Albuquerque et al., 2012; Persson and Persson, 2008).

Recently, tissue engineering has paved the way for the development of new therapeutic strategies, trying to understand and mimic the role of the cellular and matrix components of the tissues along the regenerative process. The regeneration of tissues of mesodermal origin, including periodontal tissues, rely on the use of adult mesenchymal stem cells (MSCs), which have been shown to have the capacity to differentiate into various cellular lineages (Chen and Jin, 2010; Dominici et al., 2006; Requicha et al., 2012; Tobita et al., 2008). A prime consideration in periodontal tissue engineering should be the design of three-dimensional (3D) supportive matrix/scaffolds, which will support the periodontal tissues.
Therefore, the purpose of the present study was to develop an innovative scaffold with the potential to regenerate periodontal defects. Thus, a scaffold was designed into a construct composed of two different layers: a membrane and a 3D fibre mesh, both made of a blend of starch and poly( $\varepsilon$-caprolactone) (SPCL). The membrane is expected to act as GTR barrier, avoiding gingival epithelium growth into the periodontal defect when placed between the gingiva and the defect, and also to promote the adhesion and recruitment of the native undifferentiated cells responsible for new ligament tissue formation. The SPCL 3D fibre mesh layer is aimed to provide a support matrix for the ingrowth of alveolar bone that comprises the osseous compartment of the periodontium. This fibre mesh was further functionalized with silanol groups that are known to activate bone-related gene expression and stimulate osteoblast proliferation and differentiation (Leonor et al., 2011; Ni et al., 2007; Rodrigues et al., 2012). This aforementioned double-layer scaffold was characterized in terms of morphology and surface chemical composition, mechanical properties and degradability. Furthermore, we have studied the behaviour of canine adipose-derived stem cells (cASCs) on both layers to assess their in vitro functionality.

\section{Materials and methods}

\subsection{Scaffolds preparation}

A double-layer scaffold based on SPCL, a biodegradable and thermoplastic blend of starch and poly( $\varepsilon$-caprolactone) (30:70 wt \% ; Novamont, Italy), was produced by combining two layers. These layers were obtained using two different processing techniques, solvent casting and wet-spinning, as shown in Figure 1a.

To obtain the solvent casting membrane (SPCL-M), the SPCL were dissolved in chloroform (Sigma-Aldrich, Germany) at a concentration of $20 \mathrm{wt} \%$. At room temperature, $3 \mathrm{ml}$ of the polymeric solution was cast onto a $5 \mathrm{~cm}$ diameter patterned Teflon mould to obtain the SPCL-M, which was then dried in a hood and cut into disc samples $6 \mathrm{~mm}$ in diameter.

In order to prepare the wet-spun fibre meshes, the same SPCL solution was loaded into a $5 \mathrm{ml}$ plastic syringe with a metallic needle $(21 \mathrm{G} \times 1.5$ inches $)$. The syringe was connected to a programmable syringe pump (KD Scientific, World Precision Instruments, UK) to inject the polymer solution at a controlled pumping rate of $5 \mathrm{ml} / \mathrm{h}$ to allow the formation of the fibre mesh directly into the coagulation bath. The wet-spun fibre mesh structure was formed during the process by random movement of the coagulation bath. Two different coagulation baths were used: methanol (Vaz Pereira, Portugal) as control (SPCL-WS) and calcium silicate solution (SPCL-WS-Si), previously studied and described (Leonor et al., 2011; Oyane et al., 2003; Rodrigues et al., 2012). Using methanol, the formed fibre meshes were dried at room temperature overnight in order to remove any 


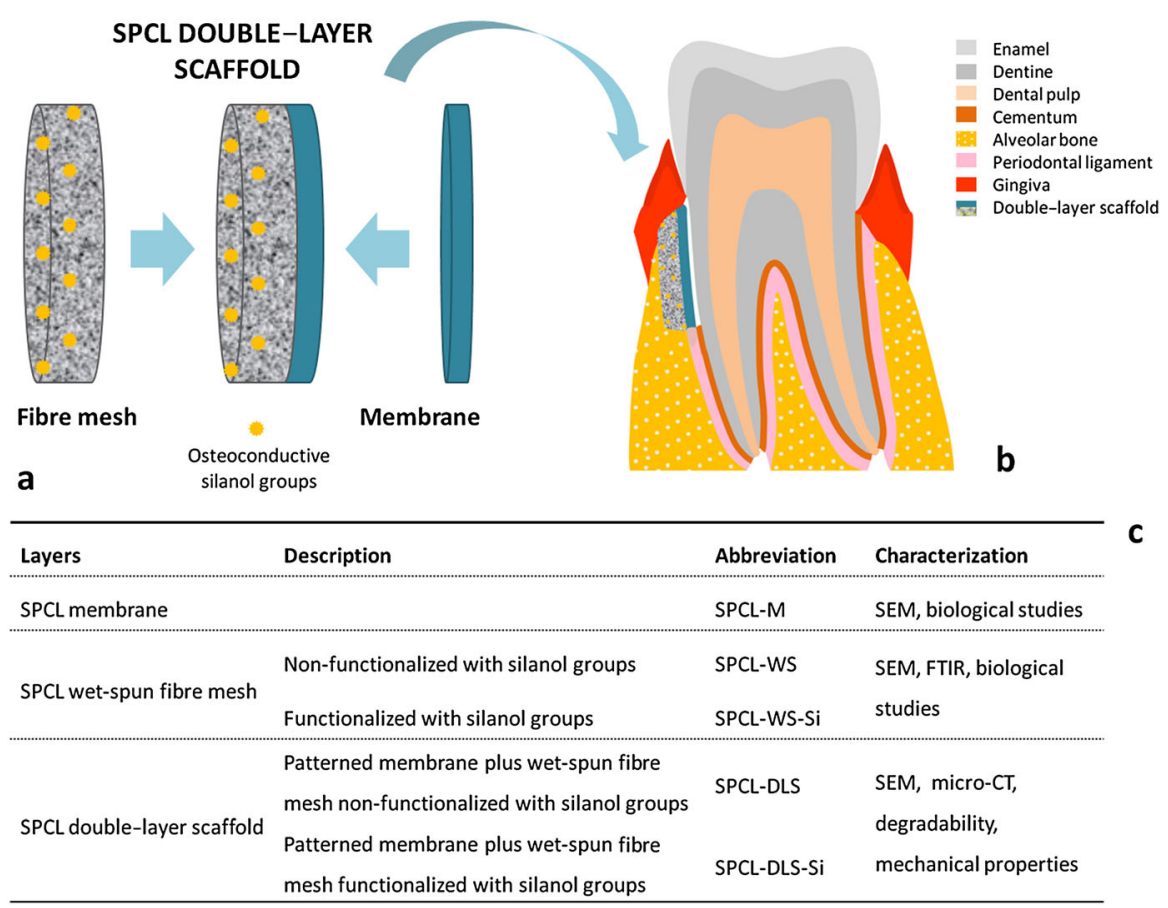

Figure 1. (a) Schematic representation of the developed double-layer scaffold comprising a membrane and a fibre mesh; yellow spots represent the osteoconductive silanol groups incorporated into the material. (b) Schematic picture of the implantation of the doublelayer scaffold in a periodontal defect. (c) Table summarizing the components of the double-layer scaffold that were developed and characterized

remaining solvent and designated as SPCL-WS. In the case of using calcium silicate solution, the fibre meshes were dried in an oven at $60^{\circ} \mathrm{C}$ for $24 \mathrm{~h}$ and designated SPCL-WS-Si.

After the preparation of each layer as described above, the two layers were combined together by dropping chloroform onto the top side of the SPCL-M, allowing it to become slightly soft and then, by applying an certain pressure, the fibre mesh was attached. The combined layers were dried in a hood and cut into discs $6 \mathrm{~mm}$ in diameter. All samples were disinfected by ethylene oxide before cell culture studies.

Figure 1c describes the composition and the characterization of the two developed layers. The double-layer scaffolds, comprising SPCL-M/SPCL-WS or SPCL-M/SPCLWS-Si, were also characterized in terms of morphology, degradability and mechanical behaviour.

\subsection{Morphology characterization}

\subsubsection{Scanning electron microscopy}

Scanning electron microscopy (SEM) was performed using a Leica Cambridge S-360 model (Cambridge, UK). Prior to any SEM observations, all the scaffolds were sputter-coated with gold by ion sputtering (Polaron SC502, Fisons Instruments, UK). Three samples of each material were used and the micrographs were analysed using ImageJ software (NIH, USA).

\subsubsection{Micro-computed tomography}

Micro-computed tomography (micro-CT) was carried out using a high-resolution micro-CT Skyscan 1072 scanner
(Skyscan, Kontich, Belgium). X-ray scans of SPCL-DLS and SPCL-DLS-Si were performed in triplicate, using a resolution of pixel size $8.7 \mu \mathrm{m}$ and integration time of $1.9 \mathrm{~s}$. The X-ray source was set at $49 \mathrm{keV}$ of energy and $122 \mu \mathrm{A}$ of current. Approximately 400 projections were acquired over a rotation range of $180^{\circ}$ with a rotation step of $0.45^{\circ}$. Datasets were reconstructed using standardized cone-beam reconstruction software (NRecon v, 1.4.3, SkyScan). Representative datasets of 200 slices were segmented into binary images with a dynamic threshold of 37-120 to identify the organic and inorganic phases. These data were used to build 3D models (CTAnalyser, v 1.5.1.5, SkyScan).

\subsection{Fourier transform attenuated total reflectance infrared spectroscopy}

The surface of the SPCL-WS and SPCL-WS-Si was analysed by Fourier transform IR spectroscopy with attenuated total reflectance (FTIR-ATR) in an IRPrestige 21 (Shimadzu, Japan). Spectra were collected at $4 \mathrm{~cm}^{-1}$ resolution, using 60 scans in the spectral range $4400-800 \mathrm{~cm}^{-1}$. For each sample, three individual measurements were performed.

\subsection{Degradation behaviour}

\subsubsection{Water uptake and weight loss}

In order to simulate in vivo conditions, SPCL-DLS and SPCL-DLS-Si were incubated in different enzymatic solutions under physiological conditions ( $\left.\mathrm{pH} 7.4,37^{\circ} \mathrm{C}\right)$. 
Preweighed materials were individually immersed in $2.5 \mathrm{ml}$ of four different solutions: (a) phosphate-buffered saline (PBS; $0.01 \mathrm{M}, \mathrm{pH} 7.4$; Sigma-Aldrich, Germany); (b) PBS solution containing $400 \mathrm{U} / 1 \alpha$-amylase from Bacillus sp. (Sigma-Aldrich, Germany); (c) PBS solution containing $500 \mathrm{U} / 1$ lipase from Pseudomonas sp. (Sigma-Aldrich, Germany); and (d) PBS containing lipase and amylase at the same concentrations (50:50 vol\%). The samples were incubated at $37^{\circ} \mathrm{C}$ for $1,3,7,14,21$ and 28 days, and the solutions were changed weekly. All the prepared solutions were sterilized using a $0.2 \mu \mathrm{m}$ syringe filter and kept at $4^{\circ} \mathrm{C}$ until further usage.

Enzymatic degradation of polymeric scaffolds was carried out using amylase and lipase, enzymes to study their effect on starch and PCL hydrolysis. Additionally, these two enzymes are found in healthy dogs' serum at concentrations in the range 373-1503 U/1 and 90-527 U/1, respectively (Tvedten, 2012). The reason for using dog reference values is based on the fact that the dog is an animal with natural occurrence of periodontal disease and is considered the best model in periodontology preclinical research.

At the end of each degradation period, samples were removed from the solution and placed between two filter papers, to remove excess of liquid, and immediately weighed to determine the water uptake. Then, it was washed several times with distilled water and placed in the oven at $37^{\circ} \mathrm{C}$ for 3 days in order to measure the dry weight and thus determine the weight loss. For each study condition (material and solution), five samples were tested. Degradation solutions were frozen for further analysis, i.e. for the quantification of the reducing sugars and calcium and silicon elements, as described below.

\subsubsection{Morphology after degradation}

SPCL-DLS and SPCL-DLS-Si samples after degradation under different conditions were dried at room temperature and observed by SEM, as described previously. Three samples of each material were analysed.

\subsection{3. $\alpha$-Amylase activity}

Degradation solutions were analysed to determine the concentration of reducing sugars released into the solution as result of starch hydrolysis. The determination was based on the dinitrosalicyclic acid (DNS) method and absorbance was read at $540 \mathrm{~nm}$ in a microplate reader (Synergy HT, BioTek Instruments, USA), using a standard curve of glucose.

\subsubsection{Calcium and silicon concentration of the degradation solutions}

Elemental concentrations of silicon ( $\mathrm{Si}$ ) and calcium (Ca) were measured in the degradation solutions after being passed through a $0.22 \mu \mathrm{m}$ filter, using inductively coupled plasma atomic emission spectrometry (ICP: JY2000-2, Jobin Yvon, Horiba, Japan).

\subsection{Mechanical behaviour}

Mechanical tests were carried out to evaluate the tensile strength of the SPCL-DLS and SPCL-DLS-Si. Five rectangular samples $(5 \times 20 \mathrm{~mm})$ were tested in the dry and wet state (after $24 \mathrm{~h}$ of immersion in PBS). Samples were tested using a uniaxial testing system (Instron 4505 Universal Machine, USA) with a load cell of $1 \mathrm{kN}$. The tensile test was carried out at a crosshead speed of $2 \mathrm{~mm} / \mathrm{min}$. Tensile stress was taken as the maximum stress in the stress-strain curve, and the elastic modulus was estimated from the initial slope of the stress-strain curve.

\subsection{Culture of canine adipose-derived stem cells}

Canine adipose-derived stem cells (cASCs) were isolated from subcutaneous abdominal tissue collected from adult healthy dogs in the Veterinary Hospital of University of Trás-os-Montes e Alto Douro, in accordance with Portuguese legislation (Portaria no 1005/92) and international standards on animal welfare, as defined by the European Directive 2010/63/EU, and with previous informed consent of the owners. cASCs were expanded in basal medium composed of Dulbecco's modified Eagle's medium (DMEM; Sigma-Aldrich, Germany) supplemented with 10\% fetal bovine serum (FBS; Invitrogen, USA) and 1\% antibiotic/ antimycotic (Sigma, USA), as previously reported (Requicha et al., 2012), until passage 2, before seeding. As previously mentioned, the dog is an important animal model in periodontal regeneration research. For that reason, we envision, ultimately, the autologous assessment of this tissue-engineered construct with canine cells in the Wikësjo model, which is considered the most adequate animal model aimed at extrapolating results for future applications in veterinary and human dentistry (Albuquerque et al., 2012; Wikesjo and Nilveus, 1991). The harvesting, isolation and characterization of the cASCs has been addressed in a previous study (Requicha et al., 2012).

The cASCs were seeded onto the SPCL-M at a concentration of $5.0 \times 10^{4}$ cells/sample and then cultured for 1 and 14 days in basal medium. Cell morphology and proliferation were characterized by SEM and DNA quantification, respectively, as described below.

The SPCL-WS and SPCL-WS-Si samples were seeded with cASCs at a concentration of $1.0 \times 10^{5}$ cells/sample and then cultured in either basal or osteogenic medium for 7 and 28 days. Osteogenic medium was composed of $\alpha$-modified Eagle's medium ( $\alpha$-MEM; Sigma-Aldrich, Germany), $10 \%$ FBS, $1 \%$ antibiotic/antimycotic and osteogenic supplements, ascorbic acid $(50 \mu \mathrm{g} / \mathrm{ml})$ (Sigma, USA), dexamethasone $\left(10^{-8} \mathrm{M}\right.$; Sigma, USA) and $\beta$-glycerophosphate (10 mm; Sigma, USA). Cell-scaffold constructs were evaluated in terms of cellular morphology and proliferation, as described below. The relative gene expression of a specific osteoblast marker, osteocalcin, was also assessed by real-time RT-PCR analysis to evaluate the 
effect of the functionalization of the polymer fibres with silanol groups in the osteogenic differentiation of cASCs.

\subsubsection{Scanning electron microscopy}

To observe the morphology of cASCs cultured onto the membrane and fibre meshes, the samples were fixed in $2.5 \%$ glutaraldehyde solution in PBS for $1 \mathrm{~h}$ at $4^{\circ} \mathrm{C}$ and then dehydrated in a series of ethanol solutions of increasing concentration (30\%, 50\%, 70\%, 90\% and 100\% v/v). Afterwards, the samples were left to dry overnight.

\subsection{2. dsDNA quantification}

A fluorimetric dsDNA quantification kit (PicoGreen, Molecular Probes, USA) was used and the fluorescence was read using a microplate ELISA reader (BioTek, USA) at an excitation of $485 / 20 \mathrm{~nm}$ and an emission of 528/ $20 \mathrm{~nm}$. The number of cells at each time point was obtained by using a previously created standard curve dsDNA content vs cASCs number.

\subsubsection{Real-time RT-PCR analysis}

For the osteocalcin gene expression analysis, the cultured materials were retrieved and kept in $800 \mu \mathrm{l}$ TRIzol reagent (Invitrogen, USA). The mRNA was extracted with TRIzol following the procedure provide by the supplier. Briefly, after an incubation of $5 \mathrm{~min}$, an additional $160 \mu \mathrm{l}$ chloroform (Sigma-Aldrich, Germany) were added; the samples were then incubated for $15 \mathrm{~min}$ at $4^{\circ} \mathrm{C}$ and centrifuged at the same temperature at $13000 \mathrm{rpm}$ for $15 \mathrm{~min}$. After centrifugation, the aqueous part was collected and an equal part of isopropanol (VWR, USA) was added. After incubation for $2 \mathrm{~h}$ at $-20^{\circ} \mathrm{C}$, the samples were washed in ethanol, centrifuged at $4^{\circ} \mathrm{C}$ at $9000 \mathrm{rpm}$ for $5 \mathrm{~min}$ and resuspended in $12 \mu \mathrm{l}$ Rase/DNase-free water (Gibco, UK). The samples were quantified using an ND1000 Spectrophotometer (NanoDrop Technologies, USA). For cDNA synthesis, samples with a 260:280 ratio of 1.7-2.0 were used. The cDNA synthesis was performed in Mastercycler real-time PCR equipment (Eppendorf, USA), using an iScript cDNA Synthesis Kit (Quanta Biosciences, USA) with an initial amount of mRNA of $2 \mu \mathrm{g}$ in a total reaction volume of $20 \mu \mathrm{l}$. RNAse-free water (Gibco, UK) was used as a negative control. After synthesis of the cDNA, real-time PCR analysis was carried out in the Mastercycler real-time PCR equipment (Eppendorf) using PerfeCta Sybr-Green FastMix (Quanta Biosciences, USA) to analyse the relative expression of the osteocalcin gene (ENSCAFT00000026668; forward GATCGTGGAAGAAGG CAAAG, reverse AGCCTCTGCCAGTTGTCTGT) in each sample, using GAPDH as the housekeeping gene (NM_001003142.1; forward CCAGAACATCATCCCTGCTT, reverse GACCACCTGGTCCTCAGTGT). The primers were designed using the Primer 3 Plus v. 0.4.0 (MWG Biotech, Germany). The $\triangle \triangle C T$ method (Livak and Schmittgen, 2011) was used, using the materials after seeding as calibrator.

\subsection{Statistical analysis}

Statistical analysis was performed using GraphPad Prism v. 5.00 (GraphPad Software Inc., USA). The statistical significance was assessed by a two-way ANOVA, followed by Bonferroni post-testing. Data are reported as mean \pm standard deviation (SD).

\section{Results}

\subsection{Morphology characterization}

The micro-CT images showed that the combination of the two different layers was effectively achieved, allowing a single asymmetric scaffold, comprising two distinct parts (Figure 2), to be obtained. SEM showed that the SPCL$\mathrm{M}$ presented an irregular and rough surface, which could be due to the textures of the mould used and the stretching of the SPCL during mechanical detachment from the mould (Figure 2). The SPCL-WS and SPCLWS-Si were mainly composed by a random arrangement of interconnected fibres. The diameter of the SPCL-WS fibres was about $192 \pm 23.5 \mu \mathrm{m}$ and SPCL-WS-Si fibres were around $195 \pm 24.6 \mu \mathrm{m}$. At higher magnifications, it was possible to observe the rough surface of the SPCL fibres, which could be due to the evaporation of chloroform. In SPCL-WS-Si, the surface of the fibres was much smoother and showed some cracks, which probably resulted from the drying process, typical of materials prepared by the sol-gel process.

\subsection{Fourier transform attenuated total reflectance infrared (FTIR-ATR) spectroscopy}

The effectiveness of the functionalization of the fibre mesh layer with $\mathrm{Si}-\mathrm{OH}$ groups was investigated by FTIR-ATR (Figure 2). Regarding the SPCL-WS, a high-intensity band at $1700-1750 \mathrm{~cm}^{-1}$ was observed for $\mathrm{C}=\mathrm{O}$, corresponding to the characteristic band of poly( $\varepsilon$-caprolactone) (Elzein et al., 2004; Pashkuleva et al., 2008). In the SPCL-WS-Si, obtained using a calcium silicate solution as a coagulation bath, the $\mathrm{C}=\mathrm{O}$ band was also detected. In addition, siloxane (Si-O-Si) reflection peaks were observed at 1020, 1080 and $1180 \mathrm{~cm}^{-1}$ and a shoulder at $1110 \mathrm{~cm}^{-1}$. A shoulder of Si-OH was observed at $960 \mathrm{~cm}^{-1}$ and a peak at $840 \mathrm{~cm}^{-1}$ for Si-OH, typically observed in silica gel (Eglin et al., 2003; Nie et al., 2005), proving the functionalization of the SPCL with silanol groups.

\subsection{Degradation behaviour}

\subsubsection{Water uptake and weight loss}

The SPCL-DLS and SPCL-DLS-Si showed different water uptake abilities with immersion time in the different degradation media used, as can be observed in Figure 3a. 

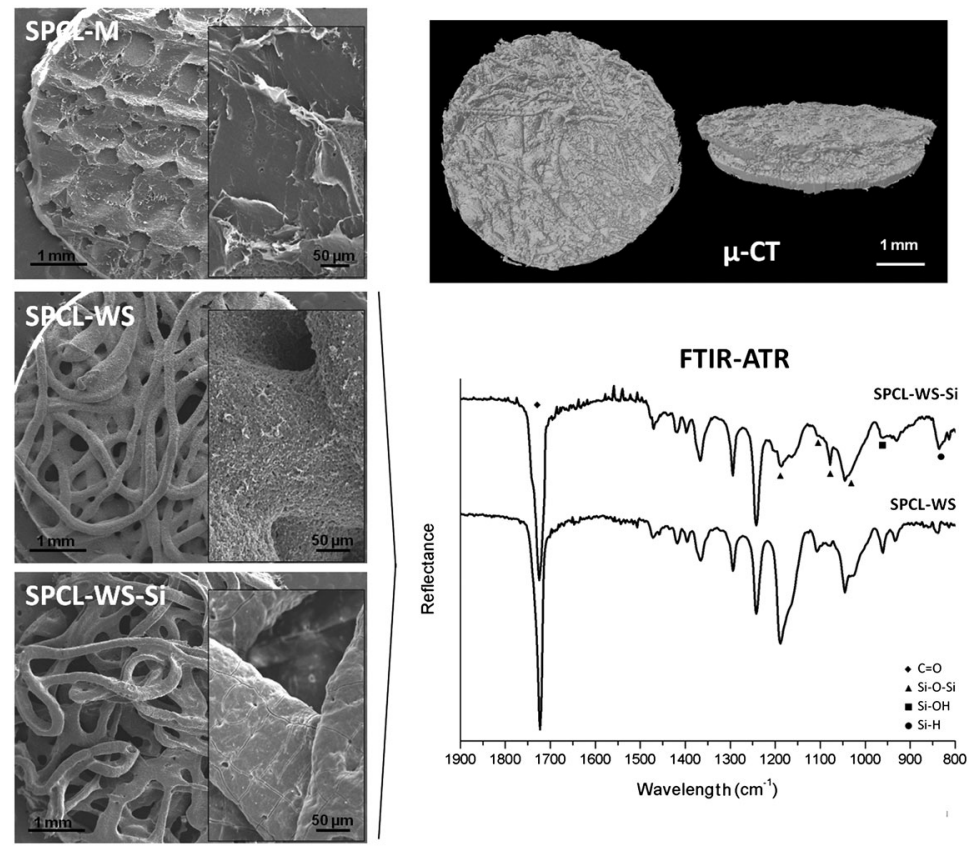

Figure 2. (Left) SEM images of SPCL-M, SPCL-WS and SPCL-WS-Si; images at the right corner of each correspond to higher magnifications. (Right) Micro-CT image of the double-layer scaffold and FTIR-ATR spectra of SPCL-WS and SPCL-WS-Si

When immersed in PBS buffer, neither scaffold changed its water uptake with immersion period, maintaining a stable value of $<60 \%$.

In contrast, scaffolds immersed in the enzymatic solutions showed a water uptake of around $50-60 \%$ after only 1 day of immersion. In the case of SPCL-DLS, a gradual increase of water uptake was observed in PBS + lipase and PBS + amylase + lipase, although it was more significant for PBS + lipase, reaching values of $96.5 \%$ compared to the $82.7 \%$ in PBS + amylase + lipase. Samples immersed in PBS + amylase solution remained constant with time. SPCL-DLSSi exhibited a lower water uptake compared to non-functionalized scaffolds. In general, the water uptake in degradation solutions increased in the following order: PBS; PBS + amylase; PBS + amylase plus lipase; and PBS + lipase.

In terms of weight loss (Figure $3 \mathrm{~b}$ ), a faster degradation of SPCL-DLS-Si than SPCL-DLS was observed after 1 day of immersion under all conditions. However, as the degradation time increased, a higher weight loss was observed for SPCL-DLS, especially at day 7 (56.9\% weight loss), in PBS + lipase. SPCL-DLS-Si, exhibited a similar degradation profile but showed a lower degradation rate in PBS + lipase (41.1\% weight loss) compared to SPCL-DLS. In PBS solution, devoid of enzymes, both materials showed a lower degradation rate.

\subsubsection{Morphology after degradation}

SEM images of the double-layer scaffolds under different enzymatic conditions as function of degradation time are presented in Figure 3c. In general, along the degradation time the structure of both scaffolds (with or without silanol groups) at the surface level showed profound morphological changes, with the formation of pores and increase of roughness being more visible in SPCL-DLS.
After day 14 of degradation in PBS + lipase and PBS + amylase + lipase, a gradual increase in the number of pores on the surface of SPCL-DLS was observed. When both scaffolds were immersed in PBS buffer, no evident changes in their morphology were possible to observe. With respect to the SPCL-DLS-Si samples, a more evident action on the polymer structure after day 14 of degradation time was also observed. The formation of calcium phosphate crystals on the surface was evident for all conditions, particularly in PBS + lipase and PBS + amylase + lipase solutions.

\subsection{3. $\alpha$-Amylase activity}

Along the degradation time, a gradual increase was observed in the concentration of sugars in the solutions for all conditions (Figure 4).

For SPCL-DLS, the amount of reducing sugars released was higher in PBS + amylase solution, particularly at day 14, indicating a high activity of this enzyme in this substrate. In the presence of lipase, although it is known that this enzyme degrades PCL-based materials, some sugars were also detected. Regarding the SPCL-DLS-Si, the presence of higher amounts of reducing sugars was also observed in PBS + amylase solution. Reducing sugars were also detected after immersion of the scaffolds in PBS, although in lower quantities. In PBS + amylase + lipase solution smaller amounts of reducing sugars were observed for both materials compared with PBS + amylase solution.

\subsubsection{Calcium and silicon concentration of the degradation solutions}

In general, an increasing release of calcium and silicon ions from the SPCL-DLS-Si was observed along the degradation time (Figure 5). Between days 3 and 14 of 
SPCL-DLS

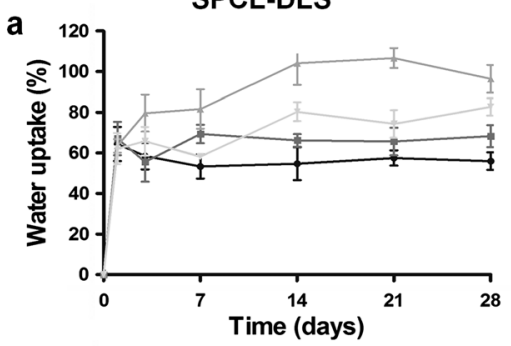

b

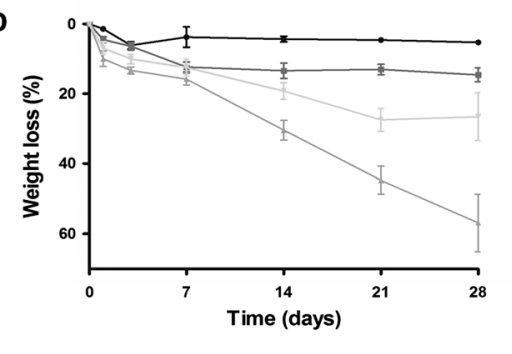

SPCL-DLS-Si
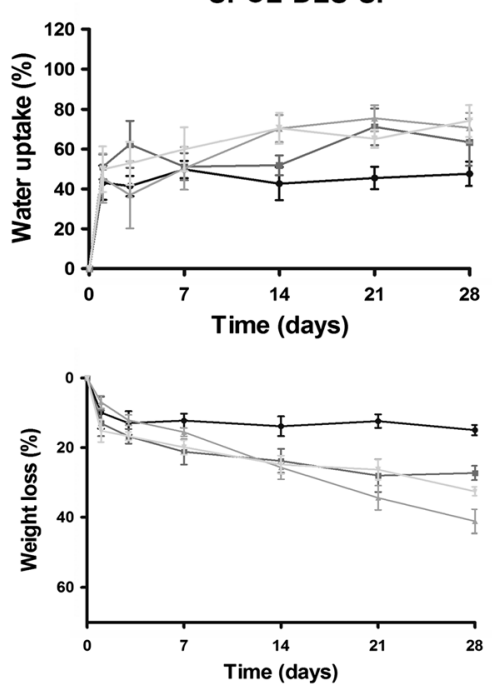

- Lipase $\neq$ Amylase + Lipase

C

SPCL-DLS

SPCL-DLS-Si

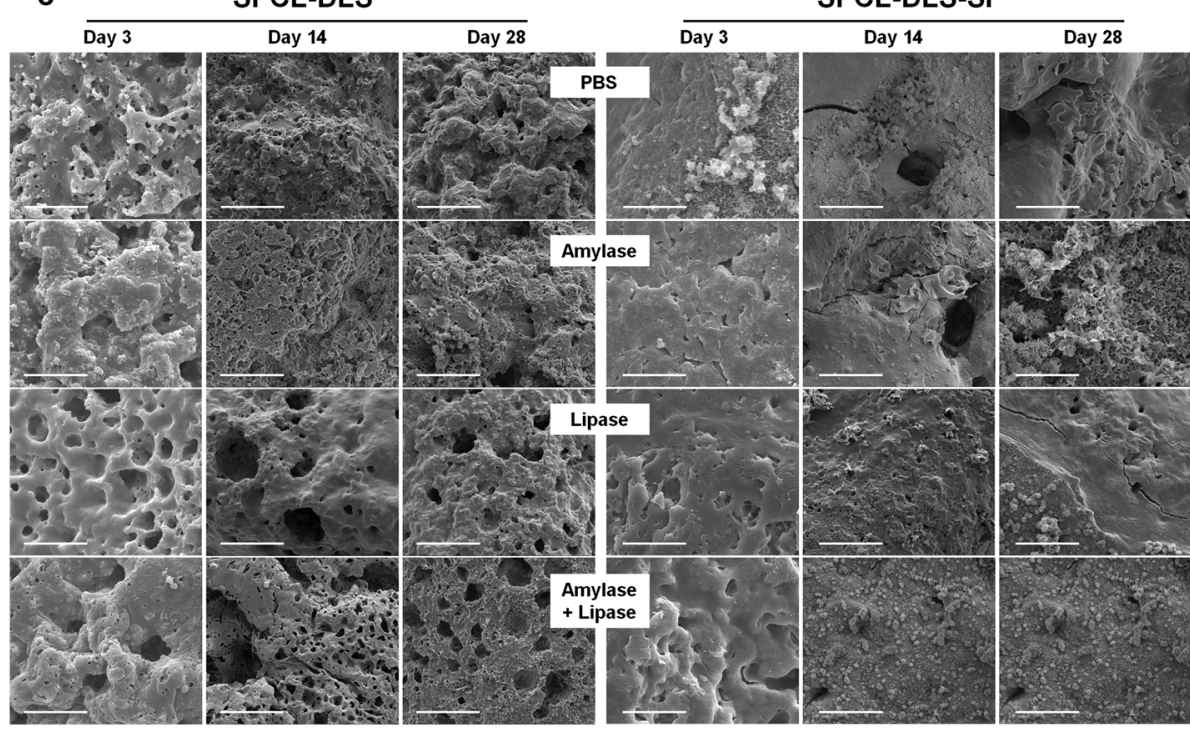

Figure 3. SEM micrographs (c) and degradation profile of the double-layer scaffolds measured in terms of water uptake (a) and weight loss (b) of SPCL-DLS and SPCL-DLS-Si in PBS solution and in the presence of enzymes under physiological conditions

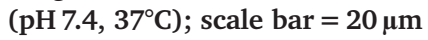
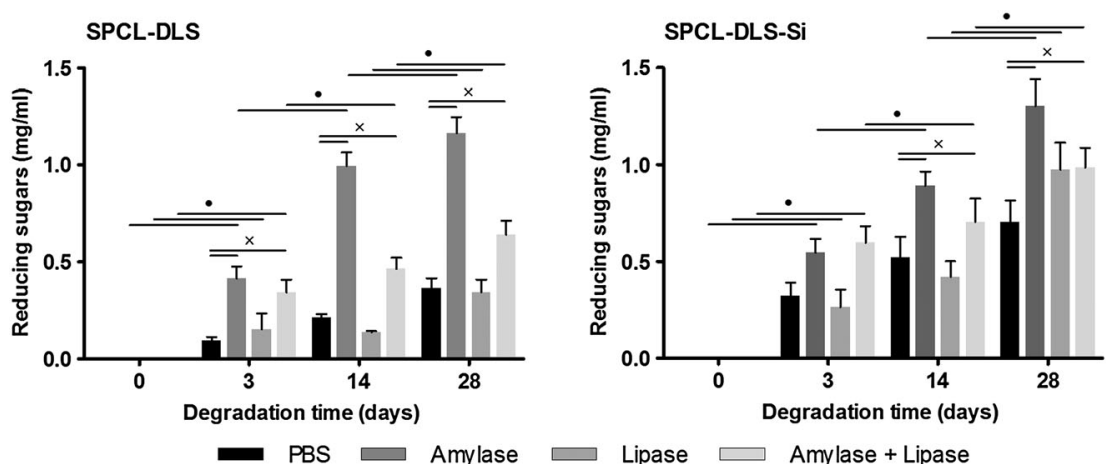

Figure 4. Cumulative release of reducing sugars as a function of degradation time in PBS solution and in the presence of enzymes under physiological conditions $\left(\mathrm{pH} 7.4,37^{\circ} \mathrm{C}\right.$ ) of SPCL-DLS and SPCL-DLS-Si: $\times$ compares the enzyme effect within the same degradation time; - compares the effect of the degradation time within the same enzymatic conditions $(p<0.001, n=9)$ 

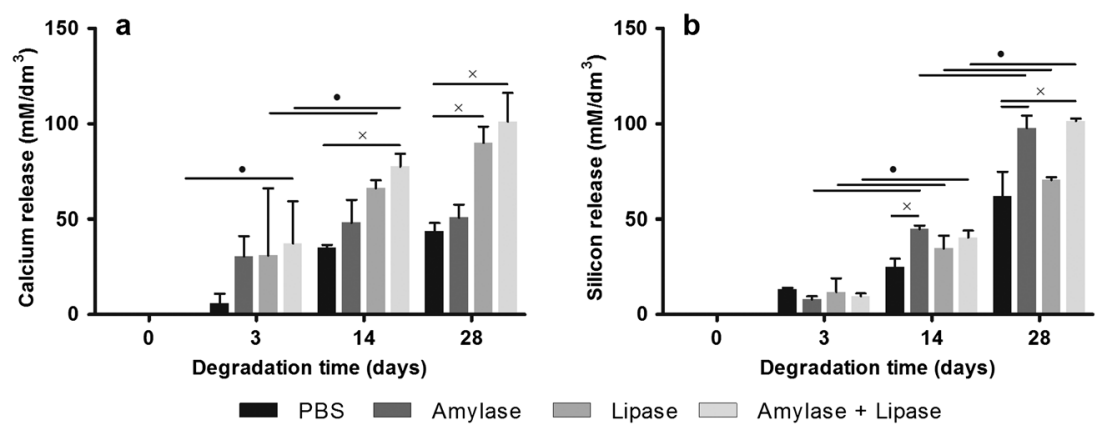

Figure 5. Cumulative release of calcium (a) and silicon (b) as function of degradation time in PBS solution and in presence of en-

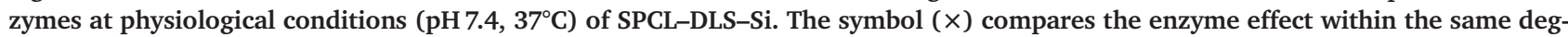
radation time. The symbol $(\bullet)$ compares the effect of the degradation time within the same enzymatic conditions $(p<0.001, n=5)$

immersion there was an increase in calcium and silicon concentration in all solutions, although the silicon concentration increase was higher. At day 14, an accentuated increase in calcium concentration was observed. The release of calcium was higher for samples immersed in PBS + lipase and PBS + amylase + lipase solutions than in PBS + amylase, while the release of silicon was much higher in samples immersed in PBS + amylase and PBS + amylase + lipase solutions.

\subsection{Mechanical behaviour}

The tensile properties of SPCL-DLS and SPCL-DLS-Si, in both wet and dry states, are reported in Table 1. In general, the mechanical properties were better for SPCL-DLS than for SPCL-DLS-Si in the dry state. In the wet state, both scaffolds present a lower modulus that is statistically different $(p<0.001)$ from samples in the dry state. In terms of elasticity, a significant increase was observed for both materials $(p<0.001)$ in the wet state.

\subsection{Culture of canine adipose-derived stem cells}

SEM images revealed good adhesion of cASCs to the SPCL$\mathrm{M}$, exhibiting a flat and elongated shape, and the cell number increase was highly significant between the first and the final culture days (Figure 6a). SEM analysis of SPCL-WS and SPCL-WS-Si revealed that, in basal medium, both materials have the ability to support and sustain cell proliferation (Figure 6b). Observation of the respective figures shows that functionalization of the polymer with silanol groups has a positive effect in cellular proliferation, evident at day 28 of culture.
The analysis of osteocalcin expression by real-time PCR (Figure 7) showed an increase of expression with culture time, higher in samples cultured in osteogenic medium. On day 28 of culture there was a significant expression of this marker in SPCL-WS-Si compared to SPCL-WS.

\section{Discussion}

This study proposed the development of an innovative double-layer scaffold that could support the regeneration of the different tissues that compose the periodontium. The developed scaffold results from the assembling of two distinct layers, a membrane obtained by solvent casting and a fibre mesh obtained by wet-spinning (Puppi et al., 2011), with different functions, aiming for the regeneration of various tissue components of a periodontal defect. This scaffold design was expected to fulfil two main goals: (a) to provide a matrix with an osteoconductive potential in one side (layer) for the formation of new alveolar bone; and (b) to simultaneously act as a barrier against the migration of gingival epithelium into the bone compartment and, at same time, to promote the formation of the PDL between bone and the tooth surface on the opposite side (layer) of the construct.

Biocompatibility and versatility of the SPCL polymeric blend were the main criteria for the selection of this material, since it has already been used to produce 3D structures and microparticles with different physicochemical characteristics using various technologies, viz. electrospinning (Martins et al., 2009a; Puppi et al., 2011), fibre bonding (Rodrigues et al., 2011), wet-spinning (Leonor et al., 2011), rapid prototyping (Martins et al., 2009a), injection moulding (Oliveira et al., 2010) and supercritical phase inversion (Duarte et al., 2010), for applications in tissue engineering

Table 1. Mechanical properties of SPCL-DLS and SPCL-DLS-Si before and after being soaked in PBS for $24 \mathrm{~h}$

\begin{tabular}{lrrr}
\hline \multicolumn{1}{c}{ Material } & SPCL-DLS dry & SPCL-DLS wet & SPCL-DLS-Si dry \\
\hline Tensile stress (MPa) & $4.08 \pm 0.877$ & $2.873 \pm 0.453$ & $3.211 \pm 1.096$ \\
Modulus (MPa) & $167.73 \pm 33.52$ & $45.839 \pm 5.38$ & $142.46 \pm 38.477$ \\
Elongation (\%) & $8.646 \pm 6.502$ & $35.854 \pm 2.925$ & $0.878 \pm 0.175$ \\
\hline
\end{tabular}



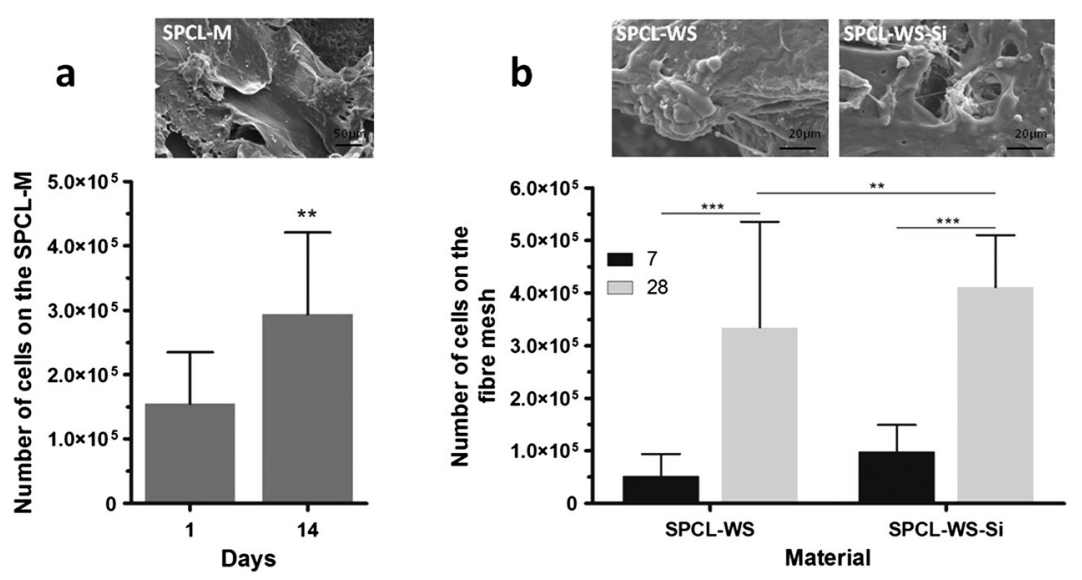

Figure 6. Canine ASCs proliferation in (a) SPCL-M cultured for 1 and 14 days and (b) SPCL-WS and SPCL-WS-Si cultured for 7 and 28 days in basal medium ( $* * p<0.01,{ }^{* * *} p<0.001, n=9$ ). SEM images represent the latter time point of each type of material

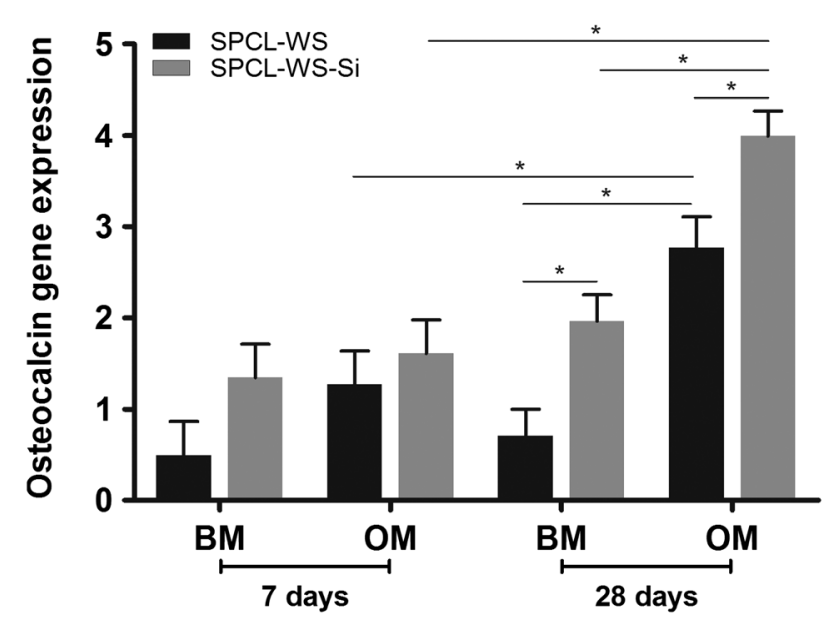

Figure 7. Osteocalcin gene expression in cASCs cultured on SPCL-WS and SPCL-WS-Si for 7 and 28 days in basal (BM) and osteogenic (OM) media $(* p<0.05, n=9)$

and regenerative medicine. Furthermore, the SPCL-based scaffolds have been extensively assessed both in vitro (Gomes et al., 2006; Puppi et al., 2011; Rodrigues et al., 2012) and in vivo (Rodrigues et al., 2011; Santos et al., 2010).

SEM and micro-CT imaging showed that the methodology used to combine the two layers did not affect the surface morphology of each layer and, at the same time, enabled solid integration of the membrane layer with the fibre mesh layer to be obtained.

The functionalization of the wet-spun fibre meshes with silanol groups, using the calcium silicate bath, was confirmed by the FTIR results. The presence of $\mathrm{Si}-\mathrm{OH}$ groups came from the hydrolysed and incomplete polycondensation reaction of TEOS, which can lead to the formation of hydrogen-bonding interactions with carbonyls of PCL, as reported previously (Eglin et al., 2003; Leonor et al., 2011; Nie et al., 2005; Rodrigues et al., 2011). In these studies, the inorganic-organic hybrids were prepared via an in situ sol-gel process of TEOS in the presence of PCL.

The functionalized fibre-mesh layer of the construct was obtained in a single step by combining wet-spinning technology and a calcium silicate solution, which allows a bioactive structure with $\mathrm{Si}-\mathrm{OH}$ functional groups to be developed. Thus, this methodology offers clear advantages in scaling-up technologies in terms of reduction of time and costs of production (Leonor et al., 2011).

Regarding the degradability of the double-layer scaffold in terms of morphology and weight loss, instead of being higher in the SPCL-DLS, a complete degradation of the studied materials was not observed, leading us to conclude that a period of 28 days of digestion by the serum enzymes is not enough to completely degrade the newly developed scaffolds.

The developed double-layer scaffold was found to be degraded by two enzymes found in dog serum ( $\alpha$-amylase and lipase) at physiological concentrations, as reported in previous studies using human-like enzymatic solutions to study their effect on the degradation of SPCL-DLS (Azevedo et al., 2003; Martins et al., 2009b). Lipase is one of the most effective enzymes in this process because it is able to catalyse the hydrolysis of ester bonds in PCL, in which, in our blend, $70 \% \mathrm{w} / \mathrm{w}$ is from PCL (Azevedo et al., 2003; Martins et al., 2009b). In PBS + amylase + lipase solution lower amounts of reducing sugars were observed for both materials compared with PBS + amylase solution, maybe because in the first solution there is a competition effect between the two enzymes.

Although, the degradation rate of the SPCL-DLS was higher in terms of weight loss compared to SPCL-DLS$\mathrm{Si}$, the production of sugars was observed to be higher in SPCL-DLS-Si, maybe influenced by the presence of silicon and calcium elements, which means that the amylase has a more important role in degrading this material than in SPCL-DLS-Si.

The gradual degradation observed for the material is essential for maintaining its integrity during the regeneration period. Along with the degradation of the material, it is expected that the pool of periodontal undifferentiated cells could be recruited, proliferated and differentiated into the desired phenotypes, both ligament and bone, at the same time that the epithelial cells of the gingiva are selectively blocked out of the defect. If the material degrades quickly, the GTR barrier function of the membrane would disappear and the previously existant cells would not colonize the defect.

Concerning the mechanical properties of SPCL-DLS and SPCL-DLS-Si, it was observed that, in general, they exhibit 
similar properties to those determined in previous studies (Ku et al., 2009) or, also, to commercially available membranes (Milella et al., 2001) for periodontal regeneration. In terms of elastic modulus, the study materials in the wet state present higher values compared to electrospun nano-apatite/PCL membrane, which could be due to the incorporation of bioceramic particles into the polymeric structure (Yang et al., 2009). It was demonstrated that a hybrid PCL membrane containing 40\% silica xerogel exhibited five and eight times higher strength and elastic modulus compared with pure PCL membrane (Lee et al., 2010).

The SPCL-DLS-Si modulus is lower than SPCL in bond states, which might be due to the bond between the carbonyl groups of PCL with silanol groups, as demonstrated in the FTIR-ATR spectrum. These bonds can cause some constraints on the mobility of the PCL chains (Eglin et al., 2003; Woodruff and Hutmacher, 2010). The mechanical properties of a scaffold for this target application should enable to maintain the space and the stability of the defect during periodontal regeneration, mainly during the early phase of wound healing (Chen and Jin, 2010). As the in vivo environment is wet, good elasticity could be advantageous for an easier siting of the scaffold in irregular defects. Moreover, the fact that SPCL-DLS-Si scaffold was not completely degraded after 28 days of immersion could be advantageous. In fact, this finding is desired because, in periodontal defects, the stability provided by the structure of the construct is very important to enable mechanical stability and prevent epithelial invasion while retaining space for regeneration.

The biological behaviour of the developed scaffold was assessed using ASCs, envisioning the future application of the constructs in tissue-engineering approaches. ASCs have revealed potential for use in tissue engineering and other cell-based therapies, similarly with bone marrow mesenchymal stem cells (BMSCs). Moreover, adipose tissue is ubiquitous and easily obtainable in large quantities, with little patient discomfort (Rada et al., 2011; Zuk et al., 2002). Canine ASCs were used to assess the studied material in terms of cellular adhesion, proliferation and osteogenic differentiation because, ultimately, the obtained tissueengineered scaffold will be assessed in a preclinical dog model, following an autologous approach. Given the fact that ASCs are similar to periodontal ligament stem cells in terms of phenotype and differentiation potential (Kato et al., 2011), they could be a suitable alternative for cell-based therapies of periodontal damage.

Both layers of the double-layer scaffold revealed potential to allow cellular adhesion and proliferation. The cultured cells exhibited a flat and elongated shape typical of mesenchymal stem cells (Dominici et al., 2006; Requicha et al., 2012; Zuk et al., 2002). In SPCL-WS and SPCL-WS-Si, the effect of functionalization with osteoconductive silanol groups in cell proliferation and osteogenic differentiation was assessed because the main aim of this layer is to enable cell attachment and promote the formation of new alveolar bone inside the periodontal defect. Although the membrane should have a barrier function, it should allow the adhesion of cells, viz. epithelial cells when placed in contact with the gingiva, or ligament cells when fitted to the tooth root surface.

The reported release of silicon in SPCL-WS-Si, as shown in previous studies (Leonor et al., 2011; Pietak et al., 2007; Reffitt et al., 2003; Rodrigues et al., 2012), induced osteogenesis, as shown by the overexpression of osteocalcin at day 28 of culture. In fact, previous studies have shown that silicon enhances osteoblast proliferation, differentiation and collagen production and also has effects on osteoclasts (Reffitt et al., 2003; Xynos et al., 2001; Zou et al., 2009). The increased release of calcium and silicon to the solution may favour some degree of reprecipitation, which, in combination with other important ions, such as phosphate, existant in stimulated buffer solution (Kokubo et al., 2009), cell culture medium (Zou et al., 2009) or tissues, can contribute to the formation of the hydroxyapatite in the material and further induce the undifferentiated cells to the osteogenic lineage (Leonor et al., 2011; Rodrigues et al., 2012), a key factor for the regeneration of the osseous compartment of the periodontium.

Considering that the scaffold is composed of two distinct layers, it was possible to obtain an asymmetric material with osteoconductive potential on one side only. This bioactive aspect was developed, aimed at being fitted on the alveolar bone defect in order to selectively promote the regeneration of this compartment of the periodontium. The porous structure of the 3D fibre mesh structure is expected to induce the proliferation of the bone cells and the integration of the scaffold in the adjacent bone tissue and ingrowth of new bone.

Depending on the type of defect, which can vary in terms of size, shape and type of damaged tissue, the scaffold might be implanted differently in order to fit adequately in the defect. It is important to place the membrane layer adjacent to the gingiva in order to avoid the migration of epithelial cells into the periodontal defect through the root surface. Moreover, when the lesion affects the radicular periodontal ligament and cementum, it is important to mould the scaffold in order to make sure that the membrane layer is adjusted to the tooth root, creating an occlusive space for regeneration of periodontal ligament, avoiding the influence of gingival epithelium migration and avoiding the risk of ankylosis with the adjacent bone.

\section{Conclusion}

This study proposed an innovative approach for designing a double-layer scaffold to address structural and biological requirements for the regeneration of periodontal defects.

The obtained data allowed us to demonstrate that this scaffold, combining the advantageous features of the two different layers in a single construct, exhibits adequate physicochemical and biological characteristics to be used in therapeutic approaches leading to the regeneration of periodontal tissues.

Therefore, this construct may provide a new solution to current clinical needs, being an alternative to mixed techniques using allograft and autograft bone combined with 
guided tissue regeneration membranes in the dentistry and orthopaedic medical fields in both human and veterinary medicine. Nevertheless, further studies, particularly using animal models, are required to demonstrate the full potential of the proposed construct in the regeneration of periodontal defects.

\section{Acknowledgements}

The research leading to these results has received funding from the European Union's Seventh Framework Programme (FP7/
2007-2013; under Grant Agreement No. REGPOT-CT2012316331-POLARIS) and from the Portuguese Foundation for Science and Technology (FCT; Grant No, MIT/ECE/0047/2009). João Requicha acknowledges the FCT for his PhD scholarship (Grant No. SFRH/BD/44143/2008).

\section{References}

Albuquerque C, Morinha F, Requicha J et al. 2012; Canine periodontitis the dog as an important model for periodontal studies. Vet J 191(3): 299-305.

Azevedo HS, Gama FM, Reis RL. 2003; In vitro assessment of the enzymatic degradation of several starch based biomaterials. Biomacromolecules 4(6): 1703-1712.

Bosshardt DD, Sculean A. 2009; Does periodontal tissue regeneration really work? Periodontology 51: 208-219.

Card SJ, Caffesse RG, Smith BA et al. 1989; New attachment following the use of a resorbable membrane in the treatment of periodontitis in dogs. Int $J$ Periodont Restor Dent 9(1): 58-69.

Chang CY, Yamada S. 2000; Evaluation of the regenerative effect of a 25\% doxycycline-loaded biodegradable membrane for guided tissue regeneration. $J$ Periodontol 71(7): 1086-1093.

Chen FM, Jin Y. 2010; Periodontal tissue engineering and regeneration: current approaches and expanding opportunities. Tissue Eng B Rev 16(2): 219-255.

Dominici M, Le Blanc K, Mueller I et al. 2006; Minimal criteria for defining multipotent mesenchymal stromal cells. The International Society for Cellular Therapy position statement. Cytotherapy 8(4): 315-317.

Duarte ARC, Mano JF, Reis RL. 2010; Supercritical phase inversion of starch-poly $(\varepsilon-$ caprolactone) for tissue engineering applications. J Mater Sci Mater Med 21(2): 533-540.

Eglin D, Ali SAM, Perry CC. 2003; A statistical study of poly( $\varepsilon$-caprolactone) crystallinity in poly( $\varepsilon$-caprolactone)-silica sol-gel materials and their in vitro calcium phosphate-forming ability. Polymer Int 52(12): 1807-1819.

Elzein T, Nasser-Eddine M, Delaite C et al. 2004; FTIR study of polycaprolactone chain organization at interfaces. $J$ Colloid Interface Sci 273(2): 381-387.

Galgut PN. 1990; Oxidized cellulose mesh used as a biodegradable barrier membrane in the technique of guided tissue regeneration. A case report. J Periodontol 61(12): 766-768.

Gomes ME, Bossano CM, Johnston CM et al. 2006; In vitro localization of bone growth factors in constructs of biodegradable scaffolds seeded with marrow stromal cells and cultured in a flow perfusion bioreactor. Tissue Eng 12(1): 177-188.

Ho MH, Hsieh CC, Hsiao SW et al. 2010; Fabrication of asymmetric chitosan GTR membranes for the treatment of periodontal disease. Carbohyd Polym 79(4): 955963.

Karring T, Nyman S, Gottlow JAN et al. 1993; Development of the biological concept of guided tissue regeneration - animal and human studies. Periodontology 2000; 1(1): 26-35.

Kato T, Hattori K, Deguchi T et al. 2011; Osteogenic potential of rat stromal cells derived from periodontal ligament. $J$ Tissue Eng Regen Med 5(10): 798-805.

Kokubo T, Matsushita T, Takadama $\mathrm{H}$ et al. 2009; Development of bioactive materials based on surface chemistry. J Eur Ceram Soc 29(7): 1267-1274.

Ku Y, Shim IK, Lee JY et al. 2009; Chitosan/ poly(L-lactic acid) multilayered membrane for guided tissue regeneration. $J$ Biomed Mater Res A 90A(3): 766-772.

Kuo L-C, Polson AM, Kang T. 2008; Associations between periodontal diseases and systemic diseases: a review of the interrelationships and interactions with diabetes, respiratory diseases, cardiovascular diseases and osteoporosis. Public Health 122(4): 417-433.

Kurtis B, Unsal B, Cetiner D et al. 2002; Effect of polylactide/glycolide (PLGA) membranes loaded with metronidazole on periodontal regeneration following guided tissue regeneration on dogs. J Periodontol 73(7): 694-700.

Lee EJ, Teng SH, Jang TS et al. 2010; Nanostructured poly( $\varepsilon$-caprolactone)silica xerogel fibrous membrane for guided bone regeneration. Acta Biomater 6(9): 3557-3565

Leonor IB, Rodrigues MT, Gomes ME et al. 2011; In situ functionalization of wet-spun fibre meshes for bone tissue engineering. J Tissue Eng Regen Med 5(2): 104-111.

Liao S, Wang W, Uo M et al. 2005; A threelayered nano-carbonated hydroxyapatite/ collagen/PLGA composite membrane for guided tissue regeneration. Biomaterials 26(36): 7564-7571

Lin NH, Gronthos S, Bartold PM. 2008; Stem cells and periodontal regeneration. Austral Dent J 53(2): 108-121.

Livak KJ, Schmittgen TD et al. 2001; Analysis of relative gene expression data using real-time quantitative PCR and the $2(\mathrm{~T})$ (-Delta Delta C) method. Methods 25(4): 402-408.

Martins A, Chung S, Pedro AJ et al. 2009a; Hierarchical starch-based fibrous scaffold for bone tissue engineering applications. $J$ Tissue Eng Regen Med 3(1): 37-42.

Martins AM, Pham QP, Malafaya PB et al. 2009b; The role of lipase and $\alpha$-amylase in the degradation of starch/poly( $\varepsilon$-caprolactone) fiber meshes and the osteogenic differentiation of cultured marrow stromal cells. Tissue Eng A 15(2): 295-305.

Milella E, Ramires PA, Brescia E et al. 2001; Physicochemical, mechanical, and biological properties of commercial membranes for GTR. J Biomed Mater Res 58(4): 427-435.

Mota J, Yu N, Caridade SG et al. 2012; Chitosan/bioactive glass nanoparticle composite membranes for periodontal regeneration. Acta Biomater 8(11): 4173-4180.

Ni S, Chang J, Chou L et al. 2007; Comparison of osteoblast-like cell responses to calcium silicate and tricalcium phosphate ceramics in vitro. $J$ Biomed Mater Res $B$ Appl Biomater 80(1): 174-183.

Nie KM, Zheng SX, Lu F et al. 2005; Inorganic-organic hybrids involving poly ( $\varepsilon$-caprolactone) and silica network: hydrogen-bonding interactions and isothermal crystallization kinetics. $J$ Polym Sci Pol Phys 43(18): 2594-2603.

Oliveira AL, Pedro AJ, Arroyo CS et al. 2010; Biomimetic Ca-P coatings incorporating bisphosphonates produced on starchbased degradable biomaterials. J Biomed Mater Res B Appl Biomater 92B(1): 55-67. Oyane A, Kawashita M, Nakanishi K et al. 2003; Bonelike apatite formation on ethylene-vinyl alcohol copolymer modified with silane coupling agent and calcium silicate solutions. Biomaterials 24(10): 1729-1735.

Park YJ, Lee YM, Park SN et al. 2000; Enhanced guided bone regeneration by controlled tetracycline release from poly (L-lactide) barrier membranes. $J$ Biomed Mater Res 51(3): 391-397.

Pashkuleva I, Azevedo HS, Reis RL. 2008; Surface structural investigation of starchbased biomaterials. Macromol Biosci 8(2): 210-219.

Persson GR, Persson RE. 2008; Cardiovascular disease and periodontitis: an update on the associations and risk. $J$ Clin Periodontol 35: 362-379.

Pietak AM, Reid JW, Stott MJ et al. 2007; Silicon substitution in the calcium phosphate bioceramics. Biomaterials 28(28): 4023-4032.

Pitaru S, Tal H, Soldinger M et al. 1989; Collagen membranes prevent apical migration 


\section{J. F. Requicha et al.}

of epithelium and support new connectivetissue attachment during periodontal wound-healing in dogs. $J$ Periodont Res 24(4): 247-253.

Puppi D, Piras AM, Chiellini F et al. 2011; Optimized electro- and wet-spinning techniques for the production of polymeric fibrous scaffolds loaded with bisphosphonate and hydroxyapatite. J Tissue Eng Regen Med 5(4): 253-263.

Quinones CR, Caton JG, Polson AM et al. 1991; Evaluation of a synthetic biodegradable barrier to facilitate guided tissue regeneration. Adv Dent Res 5(1): 85.

Rada T, Reis RL, Gomes ME. 2011; Distinct stem cells subpopulations isolated from human adipose tissue exhibit different chondrogenic and osteogenic differentiation potential. Stem Cell Rev 7(1): 64-76.

Reffitt DM, Ogston N, Jugdaohsingh R et al. 2003; Orthosilicic acid stimulates collagen type 1 synthesis and osteoblastic differentiation in human osteoblast-like cells in vitro. Bone 32(2): 127-135.

Requicha J, Viegas C, Albuquerque C et al. 2012; Effect of anatomical origin and cell passage number on the stemness and osteogenic differentiation potential of canine adipose-derived stem cells. Stem Cell Rev Rep 8(4): 1211-1222.

Rodrigues AI, Gomes ME, Leonor IB et al. 2012; Bioactive starch based scaffolds and human adipose stem cells are a good combination for bone tissue engineering. Acta Biomater 8: 3765-3776.

Rodrigues MT, Gomes ME, Viegas CA et al. 2011; Tissue-engineered constructs based on SPCL scaffolds cultured with goat marrow cells: functionality in femoral defects. J Tissue Eng Regen Med 5(1): 41-49.

Santos TC, Marques AP, Horing B et al. 2010; In vivo short-term and long-term host reaction to starch-based scaffolds. Acta Biomater 6(11): 4314-4326.

Tobita M, Uysal AC, Ogawa R et al. 2008; Periodontal tissue regeneration with adipose-derived stem cells. Tissue Eng $A$ 14(6): 945-953.

Tvedten H. 2012; Listing of selected reference values. In Small Animal Clinical Diagnosis by Laboratory Methods, Willard M, Tvedten H (eds). Elsevier Saunders: St. Louis, MO; 399-402.

Wikesjo UME, Nilveus R. 1991; Periodontal repair in dogs: healing patterns in large circumferential periodontal defects. $J$ Clin Periodontol 18(1): 49-59.

Wikesjo UME, Lim WH, Thomson RC et al. 2003; Periodontal repair in dogs: gingival tissue occlusion, a critical requirement for GTR? J Clin Periodontol 30(7): 655-664.

Woodruff MA, Hutmacher DW. 2010; The return of a forgotten polymer - polycaprolactone in the 21st century. Progr Polym Sci 35(10): 1217-1256.

Xynos ID, Edgar AJ, Buttery LDK et al. 2001; Gene-expression profiling of human osteoblasts following treatment with the ionic products of Bioglass ${ }^{\circledR} 45 S 5$ dissolution. $J$ Biomed Mater Res 55(2): 151-157.

Yang F, Both SK, Yang XC et al. 2009; Development of an electrospun nano-apatite/PCL composite membrane for GTR/GBR application. Acta Biomater 5(9): 3295-3304.

Zou S, Ireland D, Brooks RA et al. 2009; The effects of silicate ions on human osteoblast adhesion, proliferation, and differentiation. J Biomed Mater Res B Appl Biomater 90B(1): 123-130.

Zuk PA, Zhu M, Ashjian P et al. 2002 Human adipose tissue is a source of multipotent stem cells. Mol Biol Cell 13(12): 4279-4295. 